\title{
de Sitter Vacua from an Anomalous Gauge Symmetry
}

\author{
Wilfried Buchmuller, ${ }^{*}$ Markus Dierigl, ${ }^{\dagger}$ Fabian Ruehle, ${ }^{\dagger}$ and Julian Schweizer ${ }^{\S}$ \\ Deutsches Elektronen-Synchrotron DESY, 22607 Hamburg, Germany
}

(Received 10 March 2016; published 3 June 2016)

\begin{abstract}
We find a new class of metastable de Sitter solutions in compactifications of six-dimensional supergravity motivated by type IIB or heterotic string vacua. Two Fayet-Iliopoulos terms of a local U(1) symmetry are generated by magnetic flux and by the Green-Schwarz term canceling the gauge anomalies, respectively. The interplay between the induced $D$ term, the moduli dependence of the effective gauge coupling, and a nonperturbative superpotential stabilizes the moduli and determines the size of the extra dimensions.
\end{abstract}

DOI: 10.1103/PhysRevLett.116.221303

Introduction.-The observed accelerated expansion of the universe [1] is most easily explained by a constant vacuum energy density, the characteristic feature of de Sitter space. This has led to an intense search for de Sitter vacua in supergravity and superstring theories, which represent attractive extensions of the standard model of particle physics. Although much progress has been made, challenging questions still remain [2].

A particularly attractive proposal by Kachru, Kallosh, Linde, and Trivedi (KKLT) [3] is based on quantized fluxes in type IIB string theory and additional nonperturbative effects stabilizing the overall volume modulus. These features are captured in the KKLT superpotential that yields an anti-de Sitter minimum. To achieve the required "uplift" to de Sitter space additional ingredients are needed such as anti- $D 3$ branes [3,4], $D$ terms induced by magnetic flux [5-7], a gauged $R$ symmetry [8,9], or $T$-branes [10]. Alternatively, additional matter fields have to be added allowing for an $F$ term uplift (see, e.g., Refs. [11,12]). Also extensions of the KKLT proposal, such as the Large Volume Scenario [13] or Kähler Uplift [14,15] need additional ingredients to achieve an uplift to de Sitter vacua.

Conditions for de Sitter vacua in four-dimensional supergravity theories derived from string theory compactifications have previously been analyzed in Refs. [16,17]. In particular, constraints on the Kähler potential, and in the case of gauged shift symmetries, on the associated FayetIliopoulos (FI) terms, have been derived [18]. For a related discussion of de Sitter and Minkowski vacua in string theory see, for instance, Refs. [6,19-23].

In this Letter, we study de Sitter vacua in six-dimensional supergravity models, compactified on orbifolds with flux. Such models have been suggested as an intermediate step in string compactifications to four dimensions [24]. Our results represent an application of our work $[25,26]$. The crucial ingredients are the flux and the Green-Schwarz term of an anomalous local U(1) symmetry. They lead to two Fayet-Iliopoulos terms of opposite sign, and they modify the gauge kinetic function. Together with a nonperturbative KKLT-type superpotential one naturally obtains de Sitter vacua, without any additional degrees of freedom below the flux induced mass scale. In the following we derive relations between the superpotential parameters and de Sitter vacua in moduli space and give an explicit example.

Conditions for de Sitter vacua.-In this work we consider a simple $\mathcal{N}=1$ supergravity model [27] that has been derived as the effective four-dimensional Lagrangian for flux compactifications of six-dimensional supergravity $[25,26]$. It contains three chiral multiplets, $S, T, U$, and the real vector multiplet $V$ of a $\mathrm{U}(1)$ gauge symmetry. The Kähler potential is given by

$$
\begin{aligned}
K= & -\log \left(S+\bar{S}+i X^{S} V\right)-\log \left(T+\bar{T}+i X^{T} V\right) \\
& -\log (U+\bar{U}),
\end{aligned}
$$

where $X^{S, T}$ are purely imaginary and constant Killing vectors parameterizing a gauged shift symmetry. Note that the Kähler potential is of no-scale type, i.e., $K_{i} K^{i \bar{j}} K_{\bar{j}}=3$, where $i(\bar{j})$ denotes the derivative with respect to the $i$ th chiral ( $\bar{j}$ th antichiral) multiplet and $K^{i \bar{j}}$ is the inverse Kähler metric.

To completely define the gauge sector one further has to specify the gauge kinetic function $H$, whose real part $h$ corresponds to the effective gauge coupling. In the following it will be crucial that, in addition to the classical term linear in $S$, the gauge kinetic function contains a second part linear in $T$,

$$
H=h_{S} S+h_{T} T .
$$

A $T$ dependence is known to arise due to quantum corrections [29,30]. Following the convention $S=\frac{1}{2}(s+i c)$, $T=\frac{1}{2}(t+i b)$, one has $h=\frac{1}{2}\left(h_{S} s+h_{T} t\right)$. Finally, we have to specify the superpotential. It has to be gauge invariant and can therefore only depend on the linear combination

$$
Z=-i X^{T} S+i X^{S} T \equiv \frac{1}{2}(z+i \tilde{c}),
$$

where in a first step, we ignore the shape modulus $U$. For reasons discussed above we consider a KKLT-type potential, 


$$
W(Z)=W_{0}+W_{1} e^{-a Z},
$$

where, without loss of generality we choose $a>0, W_{0}$ and $W_{1}$ to be real. The third modulus $U$ can be stabilized by inclusion of a term $W_{2} \exp \left(-a^{\prime} U\right)$ in the superpotential. However, the resulting equations are considerably more involved than in the two moduli case. They will be given in Ref. [26].

In the following it will be important that the parameter $h_{T}$ in the gauge kinetic function is negative while the classical contribution $h_{S}$ is positive. Furthermore, the two Killing vectors have to be of opposite sign. As we shall see in the next section, both conditions are satisfied in flux compactifications of 6D supergravity. The line along which $h(s, t)=0$ divides the moduli space into a physical $(h>0)$ and an unphysical region $(h<0)$. The physical region thus satisfies the condition

$$
t<t_{(h)}=-\frac{h_{S}}{h_{T}} s .
$$

The scalar potential is a sum of $F$ - and $D$-term contributions,

$V=V_{F}+V_{D}=e^{K}\left(K^{i \bar{j}} D_{i} W D_{\bar{j}} \bar{W}-3|W|^{2}\right)+\frac{g^{2}}{2 h} D^{2}$,

where $D_{i} W=W_{i}+K_{i} W$ is the Kähler covariant derivative of the superpotential and $g$ is a gauge coupling, resulting in $g / \sqrt{h}$ as the effective gauge coupling. The $D$ term is given in terms of the Killing vectors,

$$
D=i K_{i} X^{i}=-\frac{i}{s} X^{S}-\frac{i}{t} X^{T} .
$$

Because of their opposite sign, $D=0$ defines a second line in $(s, t)$ space given by

$$
t_{(D)}=-\frac{X^{T}}{X^{S}} s
$$

If this line is not part of the physical region in moduli space, i.e., $\left|X^{T} / X^{S}\right| \geq\left|h_{S} / h_{T}\right|$, the $D$-term potential is positive definite. This is the situation we want to study in the following.
Solutions of the equations of motion leading to Minkowski or de Sitter vacua have to fulfill

$$
\partial_{S} V=0, \quad \partial_{T} V=0, \quad V=\epsilon \geq 0,
$$

which yield three relations between the $F$-term and $D$-term contributions to the potential. It is convenient to use the linear combinations $\partial_{ \pm}=s \partial_{S} \pm t \partial_{T}$ instead of $\partial_{S, T}$. Derivatives of the superpotential then produce the factors $\partial_{-} Z=s t D$ and $\partial_{+} Z=s t E$, where

$$
E=i K_{T} X^{T}-i K_{S} X^{S}=-\frac{i}{t} X^{T}+\frac{i}{s} X^{S} .
$$

In terms of $D$ and $E$ the scalar potential can be written in the compact form

$$
V=\frac{s t}{2}\left(D^{2}+E^{2}\right) A-E B+\frac{g^{2}}{2 h} D^{2},
$$

with

$$
\begin{aligned}
A & =\left|\partial_{Z} W\right|^{2}=a^{2} W_{1}^{2} e^{-a z}, \\
B & =2 \operatorname{Re}\left(\partial_{Z} W \bar{W}\right) \\
& =-2 a W_{1}\left(W_{1} e^{-a z}+W_{0} e^{-(a / 2) z} \cos \left(\frac{a}{2} \tilde{c}\right)\right) .
\end{aligned}
$$

Note that the term $-3|W|^{2}$ in the scalar potential Eq. (6) has disappeared due to the no-scale structure of the Kähler potential. Choosing opposite signs for $W_{0}$ and $W_{1}$, the axion $\tilde{c}$ is stabilized at zero, and in the following we set the cosine term to one. The orthogonal axion gives a mass to the U(1) vector field via the Stueckelberg mechanism [25].

In order to find minima we invert the problem and solve for the superpotential parameters in terms of $s$ and $t$. In this way we can see which superpotential parameters lead to minima in the $(s, t)$ plane for realistic parameter ranges. The conditions for extrema Eq. (9) yield three relations between the superpotential parameters and the position $(s, t)$ of the extrema in moduli space. A straightforward calculation leads to

$$
\begin{aligned}
& A=-\frac{1}{2 h^{2} s t\left(1-\rho^{2}\right)}\left[h_{T} t \rho+h\left(2-\rho+\rho^{2}\right)+h^{2} \frac{2 \epsilon}{E^{2}}\right], \\
& B=-\frac{E}{4 h^{2}\left(1-\rho^{2}\right)}\left[h_{T} t \rho\left(1+\rho^{2}\right)+h\left(2-\rho+\rho^{2}-\rho^{3}+3 \rho^{4}\right)+h^{2} \frac{8 \epsilon}{E^{2}}\right], \\
& a=-\frac{2 E\left(1-\rho^{2}\right)}{s t\left(E^{2}\left[h_{T} t \rho\left(1+\rho^{2}\right)+h\left(2-\rho+5 \rho^{2}-\rho^{3}-\rho^{4}\right)\right]+8 h^{2} \rho^{2} \epsilon\right)}\left[h_{T} t \rho+h\left(2-\rho-3 \rho^{2}\right)\right],
\end{aligned}
$$


where we have introduced the ratio $\rho=D / E \in(-1,1)$. The quantities on the rhs of these equations, $h, E$, and $\rho$, only depend on the values of the moduli fields $s$ and $t$, whereas $A, B$, and $a$ are related to the superpotential by the definitions Eq. (12) and Eq. (13).

Equations (14) can now be used to identify consistency conditions for the existence of de Sitter vacua and to construct explicit solutions. A similar discussion for pure $F$-term breaking has recently been carried out in Ref. [31]. From Eq. (12) we see that $A$ has to be positive, and by definition $a>0$. Furthermore, $B>0$ is needed for a cancellation of the positive contributions in the potential Eq. (11), thus allowing for a vanishing or small cosmological constant. From Eqs. (14) one reads off that these conditions can be satisfied if $h_{T}<0$. The reason is that the terms proportional to $h$, which would give the unwanted sign, can be made small due to the opposite sign of the two contributions $h_{S} s$ and $h_{T} t$. Given a solution for a chosen extremum at $(s, t)$, one then has to examine whether the obtained values for $h, a, W_{0}$, and $W_{1}$ are physically meaningful.

The de Sitter solutions obtained this way correspond to metastable vacua since the familiar runaway solution at infinity has zero energy density. Because of the similarity with the KKLT model one expects no further vacua. We have checked this numerically for various sets of superpotential parameters and we have indeed found no other minima. Let us finally emphasize again that the obtained Minkowski and de Sitter minima are a consequence of the opposite signs of the moduli contributions to both the gauge kinetic function and the $D$ term in combination with the nonperturbative superpotential.

In the de Sitter vacua constructed this way supersymmetry is broken by a $D$ term since, as discussed above, $D>0$ in the physical region of the moduli space. Vanishing $F$ terms, i.e., $D_{S} W=D_{T} W=0$, would imply $D \partial_{Z} W=0$, which is not possible for $D>0$ and a KKLTtype superpotential. Hence, as expected, supersymmetry is also broken by $F$ terms and the Goldstino is a mixture of the gaugino and modulini, with $m_{3 / 2} \sim\langle F\rangle \sim\langle D\rangle$. It is well known that no-scale models require carefully chosen superpotentials in order to allow for metastable de Sitter vacua $[16,17]$. In the above analysis simple solutions are found due to the gauged shift symmetry, a possibility which has already been discussed in Ref. [18].

An example.-In Ref. [26] we derive the low-energy effective action for a six-dimensional supergravity model with a U(1) gauge field and a charged bulk matter field, compactified on an orbifold $T^{2} / \mathbb{Z}_{2}$ with magnetic flux. The antisymmetric tensor field couples to the $\mathrm{U}(1)$ gauge field via a Chern-Simons term. The four-dimensional effective action involves the Kähler potential Eq. (1) with a gauged shift symmetry in $S$ and $T$. The Killing vectors are given by

$$
X^{T}=-i \frac{f}{\ell^{2}}, \quad X^{S}=-i g^{2} \frac{N+1}{(2 \pi)^{2}},
$$

where $f$ is the quantized flux, $f=-4 \pi N<0, N \in \mathbb{N}$, and $\ell$ is a length scale in Planck units. $X^{S}$ is a consequence of the Green-Schwarz term which is needed to cancel bulk and fixed point anomalies. It represents a quantum correction which, to our knowledge, has been neglected in previous analyses of moduli stabilization. Note that the two Killing vectors contribute to the $D$ term with opposite signs: $-i X^{T}>0,-i X^{S}<0$.

The orbifold compactification with $N$ flux quanta generates $N+1$ massless Weyl fermions, as discussed in Ref. [25]. Their scalar superpartners $\phi_{i}$ obtain masses from the $D$-term potential

$$
V_{D}=\frac{g^{2}}{2 h}\left(\sum_{i=1}^{N+1}\left|\phi_{i}\right|^{2}+D\right)^{2},
$$

with $D$ given by Eq. (7). The stabilization of the charged matter fields $\phi_{i}$ at the origin requires $D>0$, as assumed in the previous section. Note that all matter fields $\phi_{i}$ have the same U(1) charge since they originate from the same bulk field. The size of $D$ determines the mass splitting within matter multiplets and therefore the scale of supersymmetry breaking [32]. Furthermore, we want to point out that the quantity $E$ introduced above is always positive, $E=i X^{S} / s-i X^{T} / t>0$, and $\rho=D / E \in(0,1)$.

As discussed in the previous section, the existence of de Sitter minima crucially depends on the gauge kinetic function for which one obtains [26]

$$
h_{S}=2, \quad h_{T}=-\frac{2 g^{2} \ell^{2}}{(2 \pi)^{3}} .
$$

At the classical level, the gauge kinetic function only depends on $S$. The contribution proportional to $T$ is a direct consequence of the Green-Schwarz term, and it is remarkable that the sign of $h_{T}$ is indeed negative. Note that for $X^{T}$, $X^{S}, h_{S}$, and $h_{T}$ as given in Eqs. (15) and (17), $h>0$ always implies $D>0$ for $N \geq 1$. Hence, for nonzero flux, the matter fields are always stabilized at the origin.

In the expression Eq. (11) for the scalar potential only the term $-E B$ can become negative. For the $6 \mathrm{D}$ model considered here one has $z>0$ see Eqs. (3), (15). Hence, $B$ is bounded from below and from above, and $-E B$ can approach $-\infty$ only for $s, t \rightarrow 0$ see Eq. (10). However, in this limit the positive third term in Eq. (11) is more singular. The scalar potential is therefore bounded from below.

We are now ready to apply the general analysis of the previous section to our example. For simplicity, we set $\epsilon=0$ in Eqs. (14) and look for Minkowski vacua. Obviously, there exist very similar solutions for de Sitter vacua with $\epsilon \gtrsim 0$. Having extra dimensions at the scale of a grand unified theory (GUT) scale extra dimensions and three generations in mind [33], we choose $\ell=50, g=0.2$, and $N=3$. Using Eqs. (14) and writing $a z \equiv a_{S}(s+\kappa t$ ), we find for a Minkowski minimum at $(s, t) \simeq(5.3,9.4)$ typical superpotential parameters, 


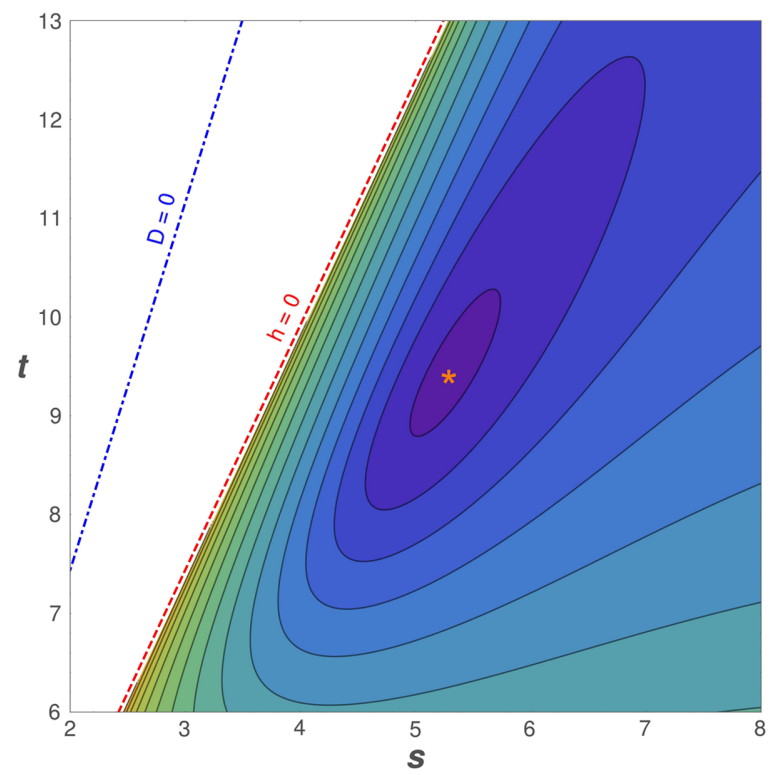

FIG. 1. Minkowski minimum for the superpotential parameters Eq. (18). $h$ vanishes along the red dashed line Eq. (5) and $D$ vanishes along the blue, dashed-dotted line Eq. (8). The minimum is at $(s, t)=(5.3,9.4)$ as indicated by the orange star.

$a_{S} \simeq 0.63, W_{0} \simeq-9.1 \times 10^{-4}, W_{1} \simeq 6.9 \times 10^{-4}$.

For the effective gauge coupling we obtain $g_{\text {eff }}=$ $g h^{-1 / 2} \simeq 0.16$, and the size of the compact dimension is $V_{2}=\ell^{2} \sqrt{s t} /\left(2 g^{2}\right)=\left(7 \times 10^{15} \mathrm{GeV}\right)^{-2}$, implying GUT scale supersymmetry breaking. Note that also larger extra dimensions can be obtained without fine-tuning for larger values of $h$, i.e., for smaller gauge couplings and smaller superpotential parameters $W_{0}, W_{1}$. The masses of all moduli are of order $10^{14} \mathrm{GeV}$. In Fig. 1 the scalar potential is shown close to the Minkowski minimum. It diverges as $s$ and $t$ approach the critical line $h=0$.

Discussion.-We have presented a new class of metastable de Sitter vacua. They arise in no-scale supergravity models with a gauged shift symmetry involving two moduli fields, $S$ and $T$. This leads to two Fayet-Iliopoulos terms with different moduli dependence. Also the gauge kinetic function has contributions linear in $S$ and $T$. The superpotential of KKLT-type depends on the gauge invariant linear combination of $S$ and $T$. As we have shown, de Sitter or Minkowski vacua are naturally obtained if the $T$-dependent contribution to the gauge kinetic function is negative.

It is remarkable that the low-energy effective action of six-dimensional supergravity with flux, compactified on an orbifold, fulfills the sufficient conditions for de Sitter vacua. The $T$-dependent FI term is generated by magnetic flux, whereas the $S$-dependent FI term and the $T$-dependent contribution to the gauge kinetic function are due to the
Green-Schwarz term needed to cancel the U(1) gauge anomaly. The Green Schwarz-term leads to the negative sign of the $T$-dependent part in the gauge kinetic function.

Flux and anomaly cancellation by the Green-Schwarz mechanism are generic ingredients of string compactifications. We therefore believe that our discussion of de Sitter vacua is relevant far beyond the six-dimensional supergravity example discussed in this Letter. It will be interesting to study applications for the heterotic string as well as for type IIB string theory and F-theory. Furthermore, it is intriguing that typical values for the superpotential parameters and the gauge coupling lead to GUT scale extra dimensions and a related large supersymmetry breaking scale. This is a challenge for the electroweak hierarchy problem, analogous to the cosmological constant problem which is encoded in the fine-tuning of the superpotential parameters.

We thank Michele Cicoli, Emilian Dudas, Stefan Groot Nibbelink, Zygmunt Lalak, Jan Louis, Hans-Peter Nilles, Alexander Westphal, and Clemens Wieck for valuable discussions. This work was supported by the German Science Foundation (DFG) within the Collaborative Research Center (SFB) 676 "Particles, Strings and the Early Universe." M. D. acknowledges support from the Studienstiftung des deutschen Volkes.

*wilfried.buchmueller@desy.de

†markus.dierigl@desy.de

fabian.ruehle@desy.de

julian.schweizer@desy.de

[1] S. Perlmutter et al. (Supernova Cosmology Project), Astrophys. J. 517, 565 (1999); A. G. Riess et al. (Supernova Search Team), Astron. J. 116, 1009 (1998).

[2] M. R. Douglas and S. Kachru, Rev. Mod. Phys. 79, 733 (2007).

[3] S. Kachru, R. Kallosh, A. D. Linde, and S. P. Trivedi, Phys. Rev. D 68, 046005 (2003).

[4] K. Choi, A. Falkowski, H. P. Nilles, and M. Olechowski, Nucl. Phys. B718, 113 (2005).

[5] C. P. Burgess, R. Kallosh, and F. Quevedo, J. High Energy Phys. 10 (2003) 056.

[6] E. Dudas and S. K. Vempati, Nucl. Phys. B727, 139 (2005).

[7] A. P. Braun, A. Hebecker, and M. Trapletti, J. High Energy Phys. 02 (2007) 015.

[8] G. Villadoro and F. Zwirner, Phys. Rev. Lett. 95, 231602 (2005).

[9] I. Antoniadis and R. Knoops, Nucl. Phys. B886, 43 (2014).

[10] M. Cicoli, F. Quevedo, and R. Valandro, J. High Energy Phys. 03 (2016) 141.

[11] O. Lebedev, H. P. Nilles, and M. Ratz, Phys. Lett. B 636, 126 (2006).

[12] R. Kallosh and A. D. Linde, J. High Energy Phys. 02 (2007) 002.

[13] V. Balasubramanian, P. Berglund, J. P. Conlon, and F. Quevedo, J. High Energy Phys. 03 (2005) 007. 
[14] V. Balasubramanian and P. Berglund, J. High Energy Phys. 11 (2004) 085.

[15] A. Westphal, J. High Energy Phys. 03 (2007) 102.

[16] L. Covi, M. Gomez-Reino, C. Gross, J. Louis, G. A. Palma, and C. A. Scrucca, J. High Energy Phys. 06 (2008) 057.

[17] L. Covi, M. Gomez-Reino, C. Gross, G. A. Palma, and C. A. Scrucca, J. High Energy Phys. 03 (2009) 146.

[18] M. Gomez-Reino and C. A. Scrucca, J. High Energy Phys. 08 (2007) 091.

[19] R. Blumenhagen, D. Lust, and T. R. Taylor, Nucl. Phys. B663, 319 (2003).

[20] G. Villadoro and F. Zwirner, J. High Energy Phys. 03 (2006) 087.

[21] M. Cicoli, S. de Alwis, and A. Westphal, J. High Energy Phys. 10 (2013) 199.

[22] A. Lukas, Z. Lalak, and E. E. Svanes, J. High Energy Phys. 08 (2015) 020.

[23] A. Retolaza and A. Uranga, J. High Energy Phys. 04 (2016) 137.
[24] A. Hebecker and M. Trapletti, Nucl. Phys. B713, 173 (2005).

[25] W. Buchmuller, M. Dierigl, F. Ruehle, and J. Schweizer, Phys. Rev. D 92, 105031 (2015).

[26] W. Buchmuller, M. Dierigl, F. Ruehle, and J. Schweizer (unpublished).

[27] We follow the conventions of Ref. [28].

[28] J. Wess and J. Bagger, Supersymmetry and Supergravity, Princeton Series in Physics (Princeton University Press, Princeton, NJ, 1992).

[29] L. E. Ibanez and H. P. Nilles, Phys. Lett. 169B, 354 (1986).

[30] L. J. Dixon, V. Kaplunovsky, and J. Louis, Nucl. Phys. B355, 649 (1991).

[31] R. Kallosh, A. Linde, B. Vercnocke, and T. Wrase, J. High Energy Phys. 10 (2014) 011.

[32] C. Bachas, arXiv:hep-th/9503030.

[33] W. Buchmuller, M. Dierigl, F. Ruehle, and J. Schweizer, Phys. Lett. B 750, 615 (2015). 